\title{
SECOND ORDER OPTIMALITY CONDITIONS FOR CONTROLS WITH CONTINUOUS AND BANG-BANG COMPONENTS
}

\author{
N.P. Osmolovskii ${ }^{1}$ and H. Maurer ${ }^{2}$ \\ ${ }^{1}$ Systems Research Institute, Polish Academy of Sciences, ul. Newelska 6, 01-447 Warszawa, \\ Poland and University of Podlasie in Siedlce,3 Maja, Siedlce,Poland, nikolai@osmolovskii.msk.ru*, \\ ${ }^{2}$ Wilhelms-Universität Münster, Institut für Numerische und Angewandte Mathematik, Einste- \\ instr. 62, D-48149 Münster, Germany, maurer@math.uni-muenster.de
}

\begin{abstract}
Second order necessary and sufficient optimality conditions for bang-bang control problems in a very general form have been obtained by the first author. These conditions require the positive (semi)-definiteness of a certain quadratic form on the finite-dimensional critical cone. In the present paper we formulate a generalization of these results to optimal control problems where the control variable has two components: a continuous unconstrained control appearing nonlinearly and a bang-bang control appearing linearly and belonging to a convex polyhedron. Many examples of control of this kind may be found in the literature.
\end{abstract}

keywords: bang-bang control, Pontryagin minimum principle, second order necessary and sufficient conditions, critical cone, quadratic form, strengthened Legendre condition

\section{Introduction}

The classical quadratic optimality conditions for an optimization or optimal control problem with constraints require that the second variation of the associated Lagrangian be nonnegative (positive) in all critical directions. Some general results along these lines were given in [5], [6], [7], [8], [9].

In the pure bang-bang case, where all control components appear linearly in the problem, second order necessary and sufficient optimality conditions have been obtained in Milyutin and Osmolovskii [4] in a very general form. In [2], [3] we have developed for this case numerical methods of testing the

*Paper partially supported by NSh-304.2003.1 and RFBR 04-01-00482.

Please use the following format when citing this chapter:

Osmolovskii, N.P., and Maurer, H., 2006, in IFIP International Federation for Information Processing, Volume 202, Systems, Control, Modeling and Optimization, eds. Ceragioli, F., Dontchev, A., Furuta, H., Marti, K., Pandolfi, L., (Boston: Springer), pp. 297-307. 
positive definiteness of the quadratic form. A different approach to second order sufficient conditions for bang-bang controls was given in [1].

In this paper we formulate both necessary and sufficient quadratic optimality conditions for optimal control problems with a control variable having two components: a continuous unconstrained control appearing nonlinearly and a bang-bang control appearing linearly and belonging to a convex polyhedron. Such a type of control problem arises in many applications. Due to space restrictions we present only a summary of results on necessary and sufficient conditions.

In Section 2, we give a statement of the general control problem with continuous and bang-bang control components (main problem). We formulate the minimum principle (first order necessary optimality condition) and the notions of a Pontryagin and a bounded-strong local minimum. In Section 3, we present second order optimality conditions, both necessary and sufficient, for these two types of minimum in the main problem.

\section{Control problem on a non-fixed time interval}

\subsection{The main problem}

Let $x(t) \in \mathbf{R}^{d(x)}$ denote the state variable and let $u(t) \in \mathbf{R}^{d(u)}, v(t) \in$ $\mathbf{R}^{d(v)}$ be the two types of control variables in the time interval $t \in\left[t_{0}, t_{1}\right]$ with a non-fixed initial time and final times $t_{0}$ and $t_{1}$. The following optimal control problem (1)-(4) will be referred to as the main problem:

Minimize $\quad \mathcal{J}\left(t_{0}, t_{1}, x(\cdot), u(\cdot), v(\cdot)\right)=J\left(t_{0}, x\left(t_{0}\right), t_{1}, x\left(t_{1}\right)\right)$

subject to the constraints

$$
\begin{array}{cl}
\dot{x}(t)=f(t, x(t), u(t), v(t)), & u(t) \in U, \quad(t, x(t), v(t)) \in \mathcal{Q}, \\
F\left(t_{0}, x\left(t_{0}\right), t_{1}, x\left(t_{1}\right)\right) \leq 0, & K\left(t_{0}, x\left(t_{0}\right), t_{1}, x\left(t_{1}\right)\right)=0, \\
\left(t_{0}, x\left(t_{0}\right), t_{1}, x\left(t_{1}\right)\right) \in \mathcal{P} . &
\end{array}
$$

The control variable $u$ appears linearly in the system dynamics,

$$
f(t, x, u, v)=a(t, x, v)+B(t, x, v) u,
$$

whereas the control variable $v$ appears nonlinearly in the dynamics in a sense which will be made more precise later. Here, $F, K, a$ are column-vector functions, $B$ is a $d(x) \times d(u)$ matrix function, $\mathcal{P} \subset \mathbf{R}^{2+2 d(x)}, \mathcal{Q} \subset \mathbf{R}^{1+d(x)+d(v)}$ are open sets and $U \subset \mathbf{R}^{d(u)}$ is a convex polyhedron. The functions $J, F, K$ are assumed to be twice continuously differentiable on $\mathcal{P}$ and the functions $a, B$ are twice continuously differentiable on $\mathcal{Q}$. The dimensions of $F, K$ are denoted by $d(F), d(K)$. By $\Delta=\left[t_{0}, t_{1}\right]$ we shall denote the interval of control. We shall use the abbreviations

$$
x_{0}=x\left(t_{0}\right), x_{1}=x\left(t_{1}\right), p=\left(t_{0}, x_{0}, t_{1}, x_{1}\right) .
$$


A process

$$
\Pi=\left\{(x(t), u(t), v(t)) \mid t \in\left[t_{0}, t_{1}\right]\right\}
$$

is said to be admissible, if $x(\cdot)$ is absolutely continuous, $u(\cdot), v(\cdot)$ are measurable bounded on $\Delta$ and the triple of functions $(x(t), u(t), v(t))$ together with the end-points $p=\left(t_{0}, x\left(t_{0}\right), t_{1}, x\left(t_{1}\right)\right)$ satisfies the constraints $(2),(3)$.

Definition 1 The process $\Pi$ affords a Pontryagin local minimum, if there is no sequence of admissible processes $\Pi^{n}=\left\{\left(x^{n}(t), u^{n}(t), v^{n}(t)\right) \mid t \in\right.$ $\left.\left[t_{0}^{n}, t_{1}^{n}\right]\right\}, n=1,2, \ldots$ such that the following properties hold with $\Delta^{n}=$ $\left[t_{0}^{n}, t_{1}^{n}\right]:$

(a) $\mathcal{J}\left(\mathrm{II}^{n}\right)<\mathcal{J}(\mathrm{II}) \quad \forall n \quad$ and $\quad t_{0}^{n} \rightarrow t_{0}, t_{1}^{n} \rightarrow t_{1}$ for $n \rightarrow \infty$;

(b) $\max _{\Delta^{n} \cap \Delta}\left|x^{n}(t)-x(t)\right| \rightarrow 0$ for $n \rightarrow \infty$;

(c) $\int_{\substack{\Delta^{n} \cap \Delta \\ \infty ;}}\left|u^{n}(t)-u(t)\right| d t \rightarrow 0, \quad \int_{\Delta^{n} \cap \Delta}\left|v^{n}(t)-v(t)\right| d t \rightarrow 0$ for $n \rightarrow$

(d) there exists a compact set $\mathcal{C} \subset \mathcal{Q}$ (which depends on the choice of the sequence) such that for all sufficiently large $n$, we have $\left(t, x^{n}(t), v^{n}(t)\right) \in$ C a.e. on $\Delta^{n}$.

For convenience, let us formulate an equivalent definition of the Pontryagin minimum.

DEFINITION 2 The process $\Pi$ affords a Pontryagin local minimum, if for each compact set $\mathcal{C} \subset \mathcal{Q}$ there exists $\varepsilon>0$ such that $\mathcal{J}\left(\Pi^{\prime}\right) \geq \mathcal{J}(\Pi)$ for all admissible processes $\Pi^{\prime}=\left\{\left(x^{\prime}(t), u^{\prime}(t), v^{\prime}(t)\right) \mid t \in\left[t_{0}^{\prime}, t_{1}^{\prime}\right]\right\}$ such that

(a) $\left|t_{0}^{\prime}-t_{0}\right|<\varepsilon, \quad\left|t_{1}^{\prime}-t_{1}\right|<\varepsilon ;$

(b) $\max _{\Delta^{\prime} \cap \Delta}\left|x^{\prime}(t)-x(t)\right|<\varepsilon$, where $\Delta^{\prime}=\left[t_{0}^{\prime}, t_{1}^{\prime}\right]$;

(c) $\int_{\Delta^{\prime} \cap \Delta}\left|u^{\prime}(t)-u(t)\right| d t<\varepsilon ; \int_{\Delta^{\prime} \cap \Delta}\left|v^{\prime}(t)-v(t)\right| d t<\varepsilon$;

(d) $\left(t, x^{\prime}(t), v^{\prime}(t)\right) \in \mathcal{C}$ a.e. on $\Delta^{\prime}$.

\subsection{First order necessary optimality conditions}

Let

$$
\Pi=\left\{(x(t), u(t), v(t)) \mid t \in\left[t_{0}, t_{1}\right]\right\}
$$

be a fixed admissible process such that the control $u(t)$ is a piecewise constant function and the control $v(t)$ is a continuous function on the interval $\Delta=$ 
$\left[t_{0}, t_{1}\right]$. In order to make the notations simpler we do not use such symbols and indices as zero, hat or asterisk to distinguish this trajectory from others. Denote by

$$
\theta=\left\{\tau_{1}, \ldots, \tau_{s}\right\}, \quad t_{0}<\tau_{1}<\ldots<\tau_{s}<t_{1}
$$

the finite set of all discontinuity points (jump points) of the control $u(t)$. Then $\dot{x}(t)$ is a piecewise continuous function whose points of discontinuity belong to $\theta$, and hence $x(t)$ is a piecewise smooth function on $\Delta$. Henceforth, we shall use the notation

$$
[u]^{k}=u^{k+}-u^{k-}
$$

to denote the jump of function $u(t)$ at the point $\tau_{k} \in \theta$, where

$$
u^{k-}=u\left(\tau_{k}-0\right), \quad u^{k+}=u\left(\tau_{k}+0\right)
$$

are the left hand and the right side hand values of the control $u(t)$ at $\tau_{k}$, respectively. Similarly, we denote by $[\dot{x}]^{k}$ the jump of the function $\dot{x}(t)$ at the point $\tau_{k}$.

Let us formulate a first-order necessary condition for optimality of the process $\Pi$ in the form of the Pontryagin minimum principle. To this end we introduce the Pontryagin or Hamiltonian function

$$
H(t, x, \psi, u, v)=\psi f(t, x, u, v)=\psi a(t, x, v)+\psi B(t, x, v) u,
$$

where $\psi$ is a row-vector of dimension $d(\psi)=d(x)$ while $x, u, f, F$ and $K$ are column-vectors. The factor of the control $u$ in the Pontryagin function will be called the switching function for the u-component

$$
\sigma(t, x, \psi, v)=\psi B(t, x, v)
$$

which is a row vector of dimension $d(u)$. Denote the end-point Lagrange function by

$$
l\left(\alpha_{0}, \alpha, \beta, p\right)=\alpha_{0} J(p)+\alpha F(p)+\beta K(p), \quad p=\left(t_{0}, x_{0}, t_{1}, x_{1}\right),
$$

where $\alpha$ and $\beta$ are row-vectors with $d(\alpha)=d(F), d(\beta)=d(K)$, and $\alpha_{0}$ is a number. We introduce a tuple of Lagrange multipliers

$$
\lambda=\left(\alpha_{0}, \alpha, \beta, \psi(\cdot), \psi_{0}(\cdot)\right)
$$

such that

$$
\psi(\cdot): \Delta \rightarrow \mathbf{R}^{d(x)}, \quad \psi_{0}(\cdot): \Delta \rightarrow \mathbf{R}^{1}
$$

are continuous on $\Delta$ and continuously differentiable on each interval of the set $\Delta \backslash \theta$. In the sequel, we shall denote first or second order partial derivatives by subscripts referring to the variables. 
Denote by $M_{0}$ the set of the normalized tuples $\lambda$ satisfying the minimum principle conditions for the process $\Pi$ :

$$
\begin{aligned}
& \alpha_{0} \geq 0, \quad \alpha \geq 0, \quad \alpha F(p)=0, \quad \alpha_{0}+\sum \alpha_{i}+\sum\left|\beta_{j}\right|=1, \\
& \dot{\psi}=-H_{x}, \quad \dot{\psi}_{0}=-H_{t} \quad \forall t \in \Delta \backslash \theta, \\
& \psi\left(t_{0}\right)=-l_{x_{0}}, \quad \psi\left(t_{1}\right)=l_{x_{1}}, \quad \psi_{0}\left(t_{0}\right)=-l_{t_{0}}, \quad \psi_{0}\left(t_{1}\right)=l_{t_{1}}, \\
& H(t, x(t), \psi(t), u, v) \leq H(t, x(t), \psi(t), u(t), v(t)) \\
& \text { for all } t \in \Delta \backslash \theta, u \in U, v \in \mathbf{R}^{d(v)} \text { such that }(t, x(t), v) \in \mathcal{Q}, \\
& H(t, x(t), \psi(t), u(t), v(t))+\psi_{0}(t)=0 \quad \forall t \in \Delta \backslash \theta .
\end{aligned}
$$

The derivatives $l_{x_{0}}$ and $l_{x_{1}}$ are taken at the point $\left(\alpha_{0}, \alpha, \beta, p\right)$, where $p=$ $\left(t_{0}, x\left(t_{0}\right), t_{1}, x\left(t_{1}\right)\right)$, while the derivatives $H_{x}, H_{t}$ are evaluated at the point $(t, x(t), \psi(t), u(t), v(t))$ for $t \in \Delta \backslash \theta$. The condition $M_{0} \neq \emptyset$ constitutes the first order necessary condition for a Pontryagin minimum of the process $\Pi$ which is the so called Pontryagin minimum principle, cf., e.g., Milyutin, Osmolovskii [4].

THEOREM 3 If the process $\Pi$ affords a Pontryagin minimum, then the set $M_{0}$ is nonempty. The set $M_{0}$ is a finite-dimensional compact set and the projector $\lambda \mapsto\left(\alpha_{0}, \alpha, \beta\right)$ is injective on $M_{0}$.

In the sequel, it will be convenient to use the simple abbreviation $(t)$ for indicating all arguments $(t, x(t), \psi(t), u(t), v(t))$, e.g., $H(t)=H(t, x(t), \psi(t), u(t), v(t))$ and $\sigma(t)=\sigma(t, x(t), \psi(t), v(t))$.

Let $\lambda=\left(\alpha_{0}, \alpha, \beta, \psi(\cdot), \psi_{0}(\cdot)\right) \in M_{0}$. From condition (11) it follows that $H(t)$ is a continuous function. In particular, we have $[H]^{k}=H^{k+}-H^{k-}=$ 0 for each $\tau_{k} \in \theta$, where $H^{k-}:=H\left(\tau_{k}, x\left(\tau_{k}\right), \psi\left(\tau_{k}\right), u\left(\tau_{k}-0\right), v\left(\tau_{k}\right)\right)$, $H^{k+}:=H\left(\tau_{k}, x\left(\tau_{k}\right), \psi\left(\tau_{k}\right), u\left(\tau_{k}+0\right), v\left(\tau^{k}\right)\right)$. We shall denote by $H^{k}$ the common value of $H^{k-}$ and $H^{k+}$ :

$$
H^{k}:=H^{k-}=H^{k+} \text {. }
$$

The equalities $[H]^{k}=0,[\psi]^{k}=0 \forall t^{k} \in \theta$ constitute the WeierstrassErdmann conditions for broken extremals. We formulate one more condition of this type which is important for the statement of second-order conditions for extremal with jumps in the control. Namely, for $\lambda \in M_{0}$ and $\tau_{k} \in \theta$ consider the function

$$
\begin{aligned}
\left(\Delta_{k} H\right)(t) & =H\left(t, x(t), \psi(t), u^{k+}, v\left(\tau_{k}\right)\right)-H\left(t, x(t), \psi(t), u^{k-}, v\left(\tau_{k}\right)\right) \\
& =\sigma\left(t, x(t), \psi(t), v\left(\tau_{k}\right)\right)[u]^{k} .
\end{aligned}
$$

LEMMA 4 For each $\lambda \in M_{0}$ the following equalities hold

$$
\left.\frac{d}{d t}\left(\Delta_{k} H\right)\right|_{t=\tau_{k}-0}=\left.\frac{d}{d t}\left(\Delta_{k} H\right)\right|_{t=\tau_{k}+0}, \quad k=1, \ldots, s .
$$


Consequently, for each $\lambda \in M_{0}$ the function $\left(\Delta_{k} H\right)(t)$ has a derivative at the point $\tau_{k} \in \theta$. Define the quantity

$$
D^{k}(H)=-\frac{d}{d t}\left(\Delta_{k} H\right)\left(\tau_{k}\right)
$$

Then the minimum condition (10) implies the following property:

LEMMA 5 For each $\lambda \in M_{0}$ the following conditions hold:

$$
D^{k}(H) \geq 0, \quad k=1, \ldots, s .
$$

The value $D^{k}(H)$ can also be written in the form

$$
\begin{aligned}
D^{k}(H) & =-H_{x}^{k+} H_{\psi}^{k-}+H_{x}^{k-} H_{\psi}^{k+}-\left[H_{t}\right]^{k} \\
& =\dot{\psi}^{k+} \dot{x}^{k-}-\dot{\psi}^{k-} \dot{x}^{k+}+\left[\psi_{0}\right]^{k},
\end{aligned}
$$

where $H_{x}^{k-}$ and $H_{x}^{k+}$ are the left- and the right-hand values of the function $H_{x}(t)$ at $\tau_{k}$, respectively, $\left[H_{t}\right]^{k}$ is the jump of the function $H_{t}(t)$ at $\tau_{k}$, etc.

\subsection{Integral cost function, unessential variables, strong minimum}

It is well known that any control problem with a cost functional in integral form

$$
\mathcal{J}=\int_{t_{0}}^{t_{1}} f_{0}(t, x(t), u(t), v(t)) d t
$$

can be brought to the canonical form (1) by introducing a new state variable $y$ defined by the state equation

$$
\dot{y}=f_{0}(t, x, u, v), \quad y\left(t_{0}\right)=0 .
$$

This yields the cost function $\mathcal{J}=y\left(t_{1}\right)$. The control variable $u$ is assumed to appear linearly in the function $f_{0}$,

$$
f_{0}(t, x, u, v)=a_{0}(t, x, v)+B_{0}(t, x, v) u .
$$

The component $y$ is called an unessential component in the augmented problem. The general definition of an unessential component is as follows.

DEFINITION 6 The state variable $x_{i}$, i.e., the $i$-th component of the state vector $x$ is called unessential if the function $f$ does not depend on $x_{i}$ and if the functions $F, J, K$ are affine in $x_{i 0}=x_{i}\left(t_{0}\right)$ and $x_{i 1}=x_{i}\left(t_{1}\right)$. 
In the following, let $\underline{x}$ denote the vector of all essential components of state vector $x$.

DEFINITION 7 We say that the process $\Pi$ affords a bounded-strong minimum if there is no sequence of admissible processes

$\Pi^{n}=\left\{\left(x^{n}(t), u^{n}(t), v^{n}(t)\right) \mid t \in\left[t_{0}^{n}, t_{1}^{n}\right]\right\}, n=1,2, \ldots$, such that

(a) $\mathcal{J}\left(\Pi^{n}\right)<\mathcal{J}(\Pi)$

(b) $t_{0}^{n} \rightarrow t_{0}, \quad t_{1}^{n} \rightarrow t_{1}, \quad x^{n}\left(t_{0}\right) \rightarrow x\left(t_{0}\right)(n \rightarrow \infty)$,

(c) $\max _{\Delta^{n} \cap \Delta}\left|\underline{x}^{n}(t)-\underline{x}(t)\right| \rightarrow 0(n \rightarrow \infty)$, where $\Delta^{n}=\left[t_{0}^{n}, t_{1}^{n}\right]$,

(d) there exists a compact set $\mathcal{C} \subset \mathcal{Q}$ (which depends on the choice of the sequence) such that for all sufficiently large $n$, we have $\left(t, x^{n}(t), v^{n}(t)\right) \in$ $\mathcal{C}$ a.e. on $\Delta^{n}$.

An equivalent definition has the form.

DEFINITION 8 The process $\Pi$ affords a bounded-strong minimum, if for each compact set $\mathcal{C} \subset \mathcal{Q}$ there exists $\varepsilon>0$ such that $\mathcal{J}\left(\Pi^{\prime}\right) \geq \mathcal{J}(\Pi)$ for all admissible processes $\Pi^{\prime}=\left\{\left(x^{\prime}(t), u^{\prime}(t), v^{\prime}(t)\right) \mid t \in\left[t_{0}^{\prime}, t_{1}^{\prime}\right]\right\}$ such that

(a) $\left|t_{0}^{\prime}-t_{0}\right|<\varepsilon, \quad\left|t_{1}^{\prime}-t_{1}\right|<\varepsilon, \quad\left|x^{\prime}\left(t_{0}\right)-x\left(t_{0}\right)\right|<\varepsilon ;$

(b) $\max _{\Delta^{\prime} \cap \Delta}\left|\underline{x}^{\prime}(t)-\underline{x}(t)\right|<\varepsilon$, where $\Delta^{\prime}=\left[t_{0}^{\prime}, t_{1}^{\prime}\right]$;

(c) $\left(t, x^{\prime}(t), v^{\prime}(t)\right) \in \mathcal{C}$ a.e. on $\Delta^{\prime}$.

The strict bounded-strong minimum is defined in a similar way, with the non-strict inequality $\mathcal{J}\left(\Pi^{\prime}\right) \geq \mathcal{J}(\Pi)$ replaced by the strict one and the process $\Pi$ ' required to be different from $\Pi$.

\section{Quadratic necessary and sufficient optimality conditions}

In this section, we shall formulate a quadratic necessary optimality condition of a Pontryagin minimum (Definition 1) for given control process $\Pi$. A strengthening of this quadratic condition yields a quadratic sufficient condition of a bounded-strong minimum (Definition 7). These quadratic conditions are based on the properties of a quadratic form on the so-called critical cone whose elements are first order variations along a given process $\Pi$. The main results of this section (Theorems 9 and 12) are due to Osmolovskii. 


\subsection{Critical cone}

For a given process II we introduce the space $\mathcal{Z}^{2}(\theta)$ and the critical cone $\mathcal{K} \subset \mathcal{Z}^{2}(\theta)$. Denote by $P_{\theta} W_{d(x)}^{1,2}\left(\Delta, \mathbf{R}^{d(x)}\right)$ the space of piecewise continuous functions $\bar{x}(\cdot): \Delta \rightarrow \mathbf{R}^{d(x)}$, which are absolutely continuous on each interval of the set $\Delta \backslash \theta$ and have a square integrable first derivative. By $L^{2}\left(\Delta, \mathbf{R}^{d(v)}\right)$ we denote the space of square integrable functions $\bar{v}(\cdot): \Delta \rightarrow \mathbf{R}^{d(v)}$. For each $\bar{x} \in P_{\theta} W_{d(x)}^{1,2}\left(\Delta, \mathbf{R}^{d(x)}\right)$ and for $\tau_{k} \in \theta$ we set

$$
\bar{x}^{k-}=\bar{x}\left(\tau_{k}-0\right), \quad \bar{x}^{k+}=\bar{x}\left(\tau_{k}+0\right), \quad[\bar{x}]^{k}=\bar{x}^{k+}-\bar{x}^{k-} .
$$

Let $\bar{z}=\left(\bar{t}_{0}, \bar{t}_{1}, \xi, \bar{x}, \bar{v}\right)$, where $\bar{t}_{0}, \bar{t}_{1} \in \mathbf{R}^{1}, \xi \in \mathbf{R}^{s}, \bar{x} \in P_{\theta} W_{d(x)}^{1,2}\left(\Delta, \mathbf{R}^{d(x)}\right)$, $\bar{v} \in L^{2}\left(\Delta, \mathbf{R}^{d(v)}\right)$. Thus,

$$
\bar{z} \in \mathcal{Z}^{2}(\theta):=\mathbf{R}^{2} \times \mathbf{R}^{s} \times P_{\theta} W_{d(x)}^{1,2}\left(\Delta, \mathbf{R}^{d(x)}\right) \times L^{2}\left(\Delta, \mathbf{R}^{d(v)}\right) .
$$

For each $\bar{z}$ we set

$$
\tilde{x}_{0}=\bar{x}\left(t_{0}\right)+\bar{t}_{0} \dot{x}\left(t_{0}\right), \quad \tilde{x}_{1}=\bar{x}\left(t_{1}\right)+\bar{t}_{1} \dot{x}\left(t_{1}\right), \quad \tilde{p}=\left(\bar{t}_{0}, \tilde{x}_{0}, \bar{t}_{1}, \tilde{x}_{1}\right) .
$$

The vector $\tilde{p}$ is considered as a column vector. Note that $\bar{t}_{0}=0$, respectively, $\bar{t}_{1}=0$ holds for a fixed initial time $t_{0}$, respectively, final time $t_{1}$. Denote by $I_{F}(p)=\left\{i \in\{1, \ldots, d(F)\} \mid F_{i}(p)=0\right\}$ the set of indices of all active endpoint inequalities $F_{i}(p) \leq 0$ at the point $p=\left(t_{0}, x\left(t_{0}\right), t_{1}, x\left(t_{1}\right)\right)$. Denote by $\mathcal{K}$ the set of all $\bar{z} \in \mathcal{Z}^{2}(\theta)$ satisfying the following conditions:

$$
\begin{aligned}
& J^{\prime}(p) \tilde{p} \leq 0, \quad F_{i}^{\prime}(p) \tilde{p} \leq 0 \forall i \in I_{F}(p), \quad K^{\prime}(p) \tilde{p}=0, \\
& \dot{\bar{x}}(t)=f_{x}^{\prime}(t, x(t), u(t), v(t)) \bar{x}(t)+f_{v}^{\prime}(t, x(t), u(t), v(t)) \bar{v}(t), \\
& {[\bar{x}]^{k}=[\dot{x}]^{k} \xi_{k}, \quad k=1, \ldots, s,}
\end{aligned}
$$

where $p=\left(x\left(t_{0}\right), t_{0}, x\left(t_{1}\right), t_{1}\right),[\dot{x}]=\dot{x}\left(\tau_{k}+0\right)-\dot{x}\left(\tau_{k}-0\right)$.

It is obvious that $\mathcal{K}$ is a convex cone with finitely many faces in the space $\mathcal{Z}^{2}(\theta)$. The convex cone $\mathcal{K}$ is called the critical cone.

\subsection{Quadratic necessary optimality conditions}

Let us introduce a quadratic form on the critical cone $\mathcal{K}$ defined by the conditions (18)-(20). For each $\lambda \in M_{0}$ and $\bar{z} \in \mathcal{K}$ we set

$$
\begin{aligned}
& \Omega(\lambda, \bar{z})=\langle A \tilde{p}, \tilde{p}\rangle+\sum_{k=1}^{s}\left(D^{k}(H) \xi_{k}^{2}-[\dot{\psi}]^{k} \bar{x}_{\mathrm{av}}^{k} \vec{\xi}_{k}\right) \\
& +\int_{t_{0}}^{t_{1}}\left(\left\langle H_{x x}(t) \bar{x}(t), \bar{x}(t)\right\rangle+2\left\langle H_{x v}(t) \bar{v}(t), \bar{x}(t)\right\rangle+\left\langle H_{v v}(t) \bar{v}(t), \bar{v}(t)\right\rangle\right) d t,(21)
\end{aligned}
$$


where

$$
\begin{aligned}
& \langle A \tilde{p}, \tilde{p}\rangle=\left\langle l_{p p} \tilde{p}, \tilde{p}\right\rangle+2 \dot{\psi}\left(t_{0}\right) \tilde{x}_{0} \bar{t}_{0}+\left(\dot{\psi}_{0}\left(t_{0}\right)-\dot{\psi}\left(t_{0}\right) \dot{x}\left(t_{0}\right)\right) \vec{t}_{0}^{2} \\
& -2 \dot{\psi}\left(t_{1}\right) \tilde{x}_{1} \bar{t}_{1}-\left(\dot{\psi}_{0}\left(t_{1}\right)-\dot{\psi}\left(t_{1}\right) \dot{x}\left(t_{1}\right)\right) \vec{t}_{1}^{2} \text {, } \\
& l_{p p}=l_{p p}\left(\alpha_{0}, \alpha, \beta, p\right), \quad p=\left(t_{0}, x\left(t_{0}\right), t_{1}, x\left(t_{1}\right)\right), \bar{x}_{\mathrm{av}}^{k}=\frac{1}{2}\left(\bar{x}^{k-}+\bar{x}^{k+}\right) \text {, } \\
& H_{x x}(t)=H_{x x}(t, x(t), \psi(t), u(t), v(t)) \text {, etc. }
\end{aligned}
$$

Note that the functional $\Omega(\lambda, \bar{z})$ is linear in $\lambda$ and quadratic in $\bar{z}$. Also note that for a problem on a fixed time interval $\left[t_{0}, t_{1}\right]$ we have $\bar{t}_{0}=\bar{t}_{1}=0$ and, hence, the quadratic form (22) reduces to $\langle A \tilde{p}, \tilde{p}\rangle=\left\langle l_{p p} \tilde{p}, \tilde{p}\right\rangle$. The following theorem gives the main second order necessary condition of optimality.

THEOREM 9 If the process $\Pi$ affords a Pontryagin minimum, then the following Condition $\mathcal{A}$ holds: the set $M_{0}$ is nonempty and

$$
\max _{\lambda \in M_{0}} \Omega(\lambda, \bar{z}) \geq 0 \text { for all } \vec{z} \in \mathcal{K} \text {. }
$$

This theorem follows from Theorem 2.3 in [7].

We call Condition $\mathcal{A}$ the necessary quadratic condition, although it is truly quadratic only if $M_{0}$ is a singleton.

\subsection{Quadratic sufficient optimality conditions}

A natural strengthening of the necessary Condition $\mathcal{A}$ turns out to be a sufficient optimality condition not only for a Pontryagin minimum, but also for a bounded-strong minimum; cf. Definition 7. Denote by $M_{0}^{+}$the set of all $\lambda \in M_{0}$ satisfying the conditions:

(a) $H(t, x(t), \psi(t), u, v)<H(t, x(t), \psi(t), u(t), v(t))$, for all $t \in \Delta \backslash \theta$, $u \in U, v \in \mathbf{R}^{d(v)}$, such that $(t, x(t), v) \in \mathcal{Q}$ and $(u, v) \neq(u(t), v(t))$;

(b) $H\left(\tau_{k}, x\left(\tau_{k}\right), \psi\left(\tau_{k}\right), u, v\right)<H^{k}$ for all $\tau_{k} \in \theta, u \in U, v \in \mathbf{R}^{d(v)}$ such that $\left(\tau_{k}, x\left(\tau_{k}\right), v\right) \in \mathcal{Q},(u, v) \neq\left(u\left(\tau_{k}-0\right), v\left(\tau_{k}\right)\right)$, $(u, v) \neq\left(u\left(\tau_{k}+0\right), v\left(\tau^{k}\right)\right)$, where $H^{k}:=H^{k-}=H^{k+}$.

Let $\operatorname{Arg} \max _{u^{\prime} \in U} \sigma u^{\prime}$ be the set of points $v \in U$ where the maximum of the linear function $\sigma u^{\prime}$ is attained

DEFINITION 10 For a given admissible process $\Pi$ with a piecewise constant control $u(t)$ and continuous control $v(t)$ we shall say that $u(t)$ is a strict bangbang control, if the set $M_{0}$ is nonempty and there exists $\lambda \in M_{0}$ such that

$$
\operatorname{Arg} \max _{u^{\prime} \in U} \sigma(t) u^{\prime}=[u(t-0), u(t+0)],
$$


where $[u(t-0), u(t+0)]$ denotes the line segment spanned by the vectors $u(t-0), u(t+0)$.

If $\operatorname{dim}(u)=1$, then the strict bang-bang property is equivalent to $\sigma(t) \neq 0$ $\forall t \in \Delta \backslash \theta$. If the set $M_{0}^{+}$is nonempty, then, obviously, $u(t)$ is a strict bang-bang control.

DEFINITION 11 An element $\lambda \in M_{0}$ is said to be strictly Legendrian if the following conditions are satisfied

(a) for each $t \in \Delta \backslash \theta$ the quadratic form

$$
\left\langle H_{v v}(t, x(t), \psi(t), u(t), v(t)) \bar{v}, \bar{v}\right\rangle
$$

is positive definite in $\mathbf{R}^{d(v)}$;

(b) for each $\tau_{k} \in \theta$ the quadratic form

$$
\left\langle H_{v v}\left(\tau_{k}, x\left(\tau_{k}\right), \psi\left(\tau_{k}\right), u\left(\tau_{k}-0\right), v\left(\tau_{k}\right)\right) \bar{v}, \bar{v}\right\rangle
$$

is positive definite in $\mathbf{R}^{d(v)}$;

(c) for each $\tau_{k} \in \theta$ the quadratic form

$$
\left\langle H_{v v}\left(\tau_{k}, x\left(\tau_{k}\right), \psi\left(\tau_{k}\right), u\left(\tau_{k}+0\right), v\left(\tau_{k}\right)\right) \bar{v}, \bar{v}\right\rangle
$$

is positive definite in $\mathbf{R}^{d(v)}$.

(d) $D^{k}(H)>0$ for all $\tau_{k} \in \theta$.

Denote by $\operatorname{Leg}_{+}\left(M_{0}^{+}\right)$the set of all strictly Legendrian elements $\lambda \in M_{0}^{+}$and put

$$
\bar{\gamma}(\bar{z})=\vec{t}_{0}^{2}+\vec{t}_{1}^{2}+\langle\xi, \xi\rangle+\left\langle\bar{x}\left(t_{0}\right), \bar{x}\left(t_{0}\right)\right\rangle+\int_{t_{0}}^{t_{1}}\langle\bar{v}(t), \bar{v}(t)\rangle d t .
$$

THEOREM 12 Let the following Condition $\mathcal{B}$ be fulfilled for the process $\Pi$ :

(a) the set $\operatorname{Leg}_{+}\left(M_{0}^{+}\right)$is nonempty;

(b) there exists a nonempty compact set

$$
M \subset \operatorname{Leg}_{+}\left(M_{0}^{+}\right)
$$

and a number $C>0$ such that

$$
\max _{\lambda \in M} \Omega(\lambda, \bar{z}) \geq C \bar{\gamma}(\bar{z}) \text { for all } \bar{z} \in \mathcal{K}
$$


Then $\Pi$ is a strict bounded-strong minimum.

If the set $\mathrm{Leg}_{+}\left(M_{0}^{+}\right)$is nonempty and $\mathcal{K}=\{0\}$ then (b) is fulfilled automatically. This is a first order sufficient optimality condition of a strict bounded-strong minimum. Let us emphasize that there is only a minimal gap between the necessary condition $\mathcal{A}$ and the sufficient condition $\mathcal{B}$ !

\section{References}

[1] A.A. Agrachev, G. Stefani, P.L. ZezzaStrong optimality for a bang-bang trajectory. SIAM J. Control and Optimization 41:991-1014, 2002.

[2] H. Maurer, N.P. Osmolovskii. Second order sufficient conditions for time optimal bangbang control problems. SIAM J. Control and Optimization 42:2239-2263, 2004.

[3] H. Maurer, N.P. Osmolovskii. Second order optimality conditions for bang-bang control problems. Control \& Cybernetics 32:555-584, 2003.

[4] A.A. Milyutin, N.P. Osmolovskii. Calculus of Variations and Optimal Control. Translations of Mathematical Monographs, Vol. 180, American Mathematical Society, Providence, 1998.

[5] N.P. Osmolovskii. High-order necessary and sufficient conditions for Pontryagin and bounded-strong minima in the optimal control problems. Dokl. Akad. Nauk SSSR, Ser. Cybernetics and Control Theory 303: 1052-1056, 1988; English transl., Sov. Phys. Dokl. 33, N. 12:883-885, 1988.

[6] N.P. Osmolovskii. Quadratic conditions for nonsingular extremals in optimal control (A theoretical treatment). Russian J. of Mathematical Physics 2:487-516, 1995.

[7] N.P. Osmolovskii. Second order conditions for broken extremal. In: Calculus of variations and optimal control. (Technion 1998), A. Ioffe, S. Reich and I. Shafir, eds., Chapman and Hall/CRC, Boca Raton, Florida, 198-216, 2000.

[8] N.P. Osmolovskii. Second-order sufficient conditions for an extremum in optimal control. Control and Cybernetics 31 803-831, 2002.

[9] N.P. Osmolovskii. Quadratic optimality conditions for broken extremals in the general problem of calculus of variations. Journal of Math. Science 123: 3987-4122, 2004. 